\title{
Validity and Sensitivity to Change of the Falls Efficacy Scales International to Assess Fear of Falling in Older Adults with and without Cognitive Impairment
}

\author{
Klaus A. Hauer ${ }^{\mathrm{a}}$ Gertrudis I.J.M. Kempen ${ }^{\mathrm{b}}$ Michael Schwenk ${ }^{\mathrm{a}} \quad$ Lucy Yardley $^{\mathrm{c}}$ \\ Nina Beyer ${ }^{d}$ Chris Todd $^{\mathrm{e}}$ Peter Oster $^{\mathrm{a}}$ G.A. Rixt Zijlstra ${ }^{\mathrm{b}}$ \\ a Bethanien-Krankenhaus/Geriatric Centre at the University of Heidelberg, Heidelberg, Germany; \\ ${ }^{b}$ CAPHRI - School for Public Health and Primary Care, Department of Health Care and Nursing Science, Maastricht \\ University, Maastricht, The Netherlands; ' School of Psychology, University of Southampton, Southampton, UK; \\ ${ }^{d}$ Musculoskeletal Rehabilitation Research Unit and Institute of Sports Medicine Copenhagen, Bispebjerg Hospital, \\ Copenhagen, Denmark; e School of Nursing, Midwifery and Social Work, University of Manchester, Manchester, UK
}

\section{Key Words}

Aged $\cdot$ Accidental falls $\cdot$ Fear of falling $\cdot$ Validation studies $\cdot$

Frail elderly $\cdot$ Cognition disorder $\cdot$ Dementia

\begin{abstract}
Background: Measures of fear of falling have not yet been validated in patients with dementia, leaving a methodological gap that limits research in a population at high risk of falling and fall-related consequences. Objective: The objectives of this study are to determine: (1) the validity of the 7-item Short Falls Efficacy Scale International (Short FES-I) in geriatric patients with and without cognitive impairment, and (2) the sensitivity to change of the 10-item Falls Efficacy Scale (FES), the 16-item FES-I and the 7-item Short FES-I in geriatric patients with dementia. Methods: Cross-sectional data of community-dwelling older adults and geriatric rehabilitation patients $(n=284)$ collected during face-to-face interviews were used to determine construct and discriminant validity by testing for differences within variables related to fear of falling. Sensitivity to change was studied in an intervention study including patients with mild to moderate de-
\end{abstract}

mentia ( $n=130$ ) as determined by standard response means (SRMs). Results: The Short FES-I showed excellent construct and discriminant validity in the total group and subsamples according to cognitive status. Sensitivity to change was adequate to good in the FES (range SRM: 0.18-0.77) and FES-I (range SRM: 0.21-0.74), with the Short FES-I showing the highest peak sensitivity to change (range SRM: 0.18-0.91). Conclusions: The Short FES-I is a valid measure to assess fear of falling in frail older adults with and without cognitive impairment, yet it may show floor effects in higher functioning older people. All scales, including the Short FES-I, were sensitive to detecting intervention-induced changes in concerns about falling in geriatric patients with dementia.

Copyright $\odot 2010$ S. Karger AG, Basel

\section{Introduction}

Falls are a major health concern in older populations and can lead to injuries, decreased physical functioning and increased health care utilization [1-4]. Falls may also induce adverse psychosocial effects such as fear of falling

\section{KARGER}

Fax +4161306 1234

E-Mail karger@karger.ch

www.karger.com
(C) 2010 S. Karger AG, Basel

0304-324X/11/0575-0462\$38.00/0

Accessible online at:

www.karger.com/ger
Dr. Klaus Hauer, PD

Bethanien-Krankenhaus/Geriatric Centre at the University of Heidelberg

Rohrbacherstrasse 149, DE-69126 Heidelberg (Germany)

Tel. +496221 319 1532, Fax +496221319 1505

E-Mail khauer@bethanien-heidelberg.de 
[4-6]. Fear of falling is common in older populations. Prevalence rates show high variability depending on various influencing factors such as the assessment method or sample selection (range: $20-85 \%$ ) with the highest rates documented in fallers and frail populations [4-8]. Fear of falling can be part of a vicious fall-associated circle as it can lead to distress and reduced quality of life, increased medication use, activity restriction, further decline in physical functioning, greater falling risk, and finally admission to institutional care $[7,9,10]$.

Older people with cognitive impairment represent a frail population with a high risk of falling and subsequent fall-related consequences which include severe injuries and increased mortality [11-14]. Yet, research on the prevention of falls in this population is limited [15] and no studies on the management of fear of falling have been performed in patients with cognitive impairment or diagnosed dementia. A major reason for this is the lack of measures validated in this specific population.

Currently existing methods to measure fear of falling apply different psychosocial constructs [16]. For prevalence studies, fear of falling has often been assessed by a single categorical questionnaire item $[17,18]$. However, there is a need for a measure which is able to discriminate between different levels of fear as well as assess concern about different types of activity. This measure could be used to (1) identify individuals with excessive fear who require intervention, (2) determine which activities cause the most fear and therefore should be targeted for rehabilitation support, and (3) accurately detect whether levels of fear have changed over time, for example following an intervention to reduce fear of falling. The concept of fear of falling has been augmented by the concept of self-efficacy or concerns about performing specific activities associated with increased risk of falling. Such concerns may be more influenced by self-perception of functional capability and less closely associated with fear and anxiety [19]. The first such scale to be developed was the Falls Efficacy Scale (FES) [20], which measures confidence in performing a range of activities of daily living (ADL) without falling. The FES is a well-established measure with acceptable reliability, validity and responsiveness (also called sensitivity to change) in different samples, i.e. community-dwelling older people, older patients in rehabilitation and older people in long-term care [16]. Yet to overcome several shortcomings of the FES, which include ceiling effects, absence of social activities, measuring confidence instead of concerns and cultural incompatibility, the Falls Efficacy Scale International (FES-I) was developed [21]. This 16-item question-

Fear of Falling Measures in People with Dementia naire is based on the original 10-item FES questionnaire and includes 6 additional items to assess concerns about falling. The FES-I extends the range of physical activities by incorporating challenging activities to prevent ceiling effects and also adds items covering social dimensions of the psychological sequelae of falls [21]. The FES-I offers a response format consisting of 4 levels ranging from 'not at all concerned' to 'very concerned', replacing the 10 categorical response format of the FES which could overtax users [21] and may lack meaningfulness for older people [22].

The FES-I showed excellent psychometric properties across community-dwelling sample populations in various countries [23]. Recently, a 7-item version of the FES-I, the Short FES-I, was developed to minimize the assessment burden and further increase acceptability. This Short FES-I also showed excellent psychometric properties in a mixed sample of community-dwelling older adults [24]. These studies have contributed to an improved assessment of concerns about falling in community-living older people, but did not specify results for different older populations. It remains unclear whether validity, reliability and sensitivity to change of these measures can be confirmed in older, more vulnerable populations such as institutionalized persons or patients with cognitive impairment. These populations in particular have been in large part excluded from previous studies because of expected methodological problems and lack of validated assessment instruments. Recently, 2 studies have reported on findings regarding the FES-I and/or Short FES-I in these populations. Hauer et al. [25] studied the feasibility, test-retest reliability and influence of administration method of the 16-item FES-I in a sample of cognitively impaired persons. Denkinger et al. [26] studied psychometric properties of the FES-I and Short FES-I in a mixed sample of institutionalized patients with no specification regarding cognitive status. Yet, knowledge on the reliability and validity of the Short FES-I in older people with cognitive impairment and the sensitivity to change of the FES-I, Short FES-I and FES in older people with dementia is still lacking.

The objective of the current study is twofold: (1) to determine the reliability and validity of the Short FES-I compared to the FES and FES-I in frail older adults with and without cognitive impairment (Study 1), and (2) to determine the sensitivity to change of the FES, FES-I and Short FES-I in patients with dementia in a randomized controlled study (Study 2). The main research question is whether existing questionnaires can be used in patients with cognitive impairment or dementia, respectively. 
The present investigation is divided into 2 parts, an observational study (Study 1: Validation) and a randomized controlled trial (RCT; Study 2: Sensitivity to Change).

We report the methods and results of both studies consecutively. Both studies were performed according to the Helsinki Declaration and were approved by the ethics committee of the local university.

\section{Study 1: Validation}

\section{Methods}

Participants

Participants $(n=284)$, comprising cognitively impaired and cognitively intact people, were consecutively recruited from 2 geriatric rehabilitation wards in Heidelberg and Stuttgart, Germany, between June 2005 and March 2008. Inclusion criteria were age $\geq 65$ years, no severe psychological or somatic disease that would prevent participants from taking part in the study, ability to walk $10 \mathrm{~m}$ without the use of a walking aid and written informed consent. Additional written informed consent was obtained from legal representatives of patients with cognitive impairment. Study participants with Mini-Mental State Examination (MMSE) scores <24 (range: 17-23) were considered cognitively impaired and participants with scores $\geq 24$ (range: $24-30$ ) were considered cognitively intact [27].

\section{Measures and Data Collection}

Concerns about falling were measured by the 16-item FES-I $[21,23]$, which was developed as part of the work of the Prevention of Falls Network Europe (www.profane.eu.org) [28]. The 4 response options for the scales range from 'not at all concerned' to 'very concerned'. Detailed information on the items, their factorial structure, the development of the FES-I and Short FES-I, and their validation in community-dwelling older people has been published [21, 23, 24]. The FES [20] consists of 10 items (items 1-10) and the Short FES-I consists of 7 items (items 2, 4, 6, 7, 9, 15 and 16) from the 16-item FES-I [21, 24].

The following data were collected in order to describe the sample and to study construct and discriminant validity: age, gender, education (number of years of educational and professional training), overall fear of falling (single-item question with answer options 'not at all', 'a little', 'quite a bit' and 'very much') [29], activity of daily living (Barthel ADL-Index) [30], timed up-and-go [31], number of diagnoses, number of medications, level of independence (autonomous, supported or institutionalized), cognitive status (MMSE) and number of falls in the previous year. Age, social status, number of diagnoses and medications were retrieved from standardized patient reports at admission. Medication or complementary agents that were not prescribed in the hospital (e.g. homeopathic agents or vitamins) were not documented. All diagnoses listed in the discharge report were documented.

Items for construct validity were selected according to previous validation studies to ensure comparability of results to persons without cognitive impairment as assessed in these studies [21, 23-25]. All items used to test construct validity such as age, sex, dizziness, cognitive status, history of falls, motor performance, functional performance, report of fear of falling, number of medications and comorbidity represent risk factors for falls [32-35].

Data were collected during patient interviews or standardized testing by trained interviewers and assessors. An interview-based administration method was used to minimize missing data as documented for self-report data [25].

\section{Statistical Analysis}

Differences between subgroups were analyzed by $\chi^{2}$ and unpaired t tests, as well as the Mann-Whitney U test, the latter in the case of non-normally distributed data. Reliability statistics for FES, FES-I and Short FES-I were computed by Cronbach's $\alpha$ (internal consistency reliability) and mean inter-item correlations. Cronbach's $\alpha>0.90$ can be considered excellent, $>0.80$ good, $>0.70$ acceptable, $>0.60$ questionable, $>0.50$ poor and $<0.50$ unacceptable [36]. Associations between the FES, FES-I, Short FESI and overall fear of falling (single question) were analyzed by Spearman's rank-order correlation test. To test for construct validity, mean scores and standard deviations for the scales were computed for the total and subgroups of cognitively impaired and cognitively intact patients according to several fall risk parameters that are associated with fear of falling. Differences between groups were tested with Mann-Whitney U, $\chi^{2}$ and Kruskal-Wallis tests. Statistical analyses were performed with SPSS for Windows, Version 17.0 (SPSS Inc., Chicago, Ill., USA).

\section{Results}

Participants

The total sample comprised 284 participants: 157 persons with and 127 persons without cognitive impairment. Descriptive data on age, functional performance, comorbidity and history of falls are consistent with a frail older population. No statistically significant differences in descriptive characteristics were revealed in the participant groups according to cognitive impairment except for the group defining variable, i.e. cognitive status (MMSE; table 1).

Descriptives and Reliability of the FES, FES-I and Short

FES-I by Cognitive Impairment

Descriptives and reliability estimates for the 3 scales are presented in table 2. For the total group, the mean FES score at baseline was 17.1 (SD: 6.7; range: 10-39), the mean FES-I score was 29.4 (SD: 10.5; range: 16-63) and the mean score of the Short FES-I was 12.8 (SD: 4.9; range: 7-28). Subgroups of patients with and without cognitive impairment did not differ significantly with respect to FES, FES-I and Short FES-I. For all scales a limited floor effect was shown, i.e. some participants reported no concerns about falling. The floor effect was smallest for the FES-I at 3.9\% $(n=11)$ compared to $12.7 \%(n=36)$ for the Short FES-I and $13.4 \%(n=38)$ for the FES. No relevant ceiling effects were detected; only 1 participant with cognitive impairment reached the maximum score on the Short FES-I.

Internal consistency was excellent for the FES-I (0.92) and good for the FES (0.89) and Short FES-I (0.84). Mean inter-item correlations for the FES, FES-I and Short FES-I were $0.45,0.43$ and 0.43 , respectively. Correlations and Cronbach's $\alpha$ estimates were slightly higher in the group without cognitive impairment (table 2). 
Table 1. Baseline characteristics of the population in the validation study (Study 1$)^{\mathrm{a}}$

\begin{tabular}{|c|c|c|c|}
\hline Variable & $\begin{array}{l}\text { Cognitively } \\
\text { impaired } \\
(\mathrm{n}=157)^{\mathrm{b}}\end{array}$ & $\begin{array}{l}\text { Cognitively } \\
\text { intact } \\
(\mathrm{n}=127)^{\mathrm{b}}\end{array}$ & $\mathrm{p}$ \\
\hline Age, years & $82.5 \pm 6.2$ & $81.6 \pm 6.8$ & $0.23^{c}$ \\
\hline \multicolumn{4}{|l|}{ Sex, n (\%) } \\
\hline Male & $38(24.2)$ & $38(29.9)$ & \multirow[t]{2}{*}{$0.28^{\mathrm{d}}$} \\
\hline Female & $119(75.8)$ & $89(70.1)$ & \\
\hline Years of education & $10.9 \pm 2.9$ & $11.5 \pm 2.8$ & $0.10^{c}$ \\
\hline Cognitive status (MMSE) & $20.6 \pm 2.3$ & $26.4 \pm 1.8$ & $<0.001^{\mathrm{c}}$ \\
\hline \multicolumn{4}{|c|}{ Level of independence, $\mathrm{n}(\%)$} \\
\hline Autonomous & $66(42.0)$ & $70(55.1)$ & \multirow[t]{3}{*}{$0.10^{\mathrm{d}}$} \\
\hline With support & $80(51.0)$ & $48(37.8)$ & \\
\hline Institutionalized & $11(7.0)$ & $9(7.1)$ & \\
\hline \multicolumn{4}{|l|}{ Dizziness, n (\%) } \\
\hline No dizziness & $77(49.4)$ & $58(46.4)$ & \multirow[t]{2}{*}{$0.62^{\mathrm{d}}$} \\
\hline Dizziness & $79(50.6)$ & $67(53.6)$ & \\
\hline Medical diagnosis, $\mathrm{n}$ & $7.3 \pm 3.0$ & $7.1 \pm 2.5$ & $0.57^{\mathrm{c}}$ \\
\hline Medications, $\mathrm{n}$ & $7.0 \pm 3.2$ & $7.0 \pm 3.0$ & $0.94^{\mathrm{c}}$ \\
\hline \multicolumn{4}{|l|}{ Falls in previous year, $\mathrm{n}(\%)$} \\
\hline No falls & $59(38.1)$ & $43(33.9)$ & \multirow[t]{3}{*}{$0.58^{\mathrm{d}}$} \\
\hline 1 fall & $37(23.9)$ & $36(28.3)$ & \\
\hline$>1$ fall & $59(38.1)$ & $48(37.8)$ & \\
\hline Activities of daily living ${ }^{\mathrm{e}}$ & $75.6 \pm 17.8$ & $78.8 \pm 17.1$ & $0.13^{c}$ \\
\hline Timed up-and-go, $\mathrm{s}^{\mathrm{f}}$ & $20.9 \pm 14.4$ & $19.9 \pm 13.0$ & $0.55^{\mathrm{c}}$ \\
\hline \multicolumn{4}{|l|}{ Fear of falling, n (\%) } \\
\hline Not at all & $75(47.8)$ & $46(36.2)$ & \multirow[t]{4}{*}{$0.24^{\mathrm{d}}$} \\
\hline A little & $54(34.4)$ & $50(39.4)$ & \\
\hline Quite a bit & $17(10.8)$ & $21(16.5)$ & \\
\hline Very much & $11(7.0)$ & $10(7.9)$ & \\
\hline
\end{tabular}

Values are means \pm SD unless otherwise indicated.

${ }^{a}$ Cognitively impaired: MMSE score <24; cognitively intact: MMSE score $\geq 24$.

${ }^{b}$ Numbers may not add up to 157 or 127 due to missing values.

${ }^{c} t$ test to test for differences between the cognitively impaired and cognitively intact group.

${ }^{\mathrm{d}} \chi^{2}$ to test for differences between the cognitively impaired and cognitively intact group.

e Higher scores indicate higher functional level.

${ }^{\mathrm{f}}$ Lower scores indicate better functional performance.

Validity

FES, FES-I and Short FES-I were significantly correlated for the total group (FES $\cdot$ FES-I Spearman's $\rho\left(r_{S}\right)=0.97$, FES $\cdot$ Short FES-I $r_{\mathrm{S}}=0.95$ and FES-I $\cdot$ Short FES-I $r_{\mathrm{S}}=0.96$; $\left.\mathrm{p}<0.01\right)$ and for each subgroup classified according to cognitive status (range $r_{\mathrm{S}}$ : $0.95-0.97$; $<<0.01$ ). Spearman correlations between the overall single-item fear of falling question and each falls efficacy scale were also significant and ranged from 0.48 to $0.51(\mathrm{p}<0.01)$ for the total group, from 0.45 to $0.50(\mathrm{p}<0.01)$ for the subgroup with cognitive impairment and from 0.50 to $0.53(\mathrm{p}<0.01)$ for the subgroup without cognitive impairment (not tabulated).
Table 2. Scoring and reliability estimates of the FES, FES-I and Short FES-I at baseline for cognitively impaired and cognitively intact older adults ${ }^{\mathrm{a}}$

\begin{tabular}{|c|c|c|c|}
\hline & $\begin{array}{l}\text { Cognitively } \\
\text { impaired } \\
(\mathrm{n}=157)\end{array}$ & $\begin{array}{l}\text { Cognitively } \\
\text { intact } \\
(\mathrm{n}=127)\end{array}$ & $\mathrm{p}$ \\
\hline \multicolumn{4}{|c|}{ Mean score $(\mathrm{SD})^{\mathrm{e}}$} \\
\hline FES & $17.0(6.7)$ & $17.1(6.8)$ & $0.87^{\mathrm{b}}$ \\
\hline FES-I & $29.2(10.3)$ & $29.6(10.7)$ & $0.75^{\mathrm{b}}$ \\
\hline Short FES-I & $12.7(4.9)$ & $12.9(5.1)$ & $0.72^{\mathrm{b}}$ \\
\hline \multicolumn{4}{|l|}{ Median (range) } \\
\hline FES & $15.0(10-39)$ & $15.0(10-36)$ & $0.99^{\circ}$ \\
\hline FES-I & $26.0(16-63)$ & $26.0(16-59)$ & $0.84^{\mathrm{c}}$ \\
\hline Short FES-I & $12.0(7-28)$ & $11.0(7-26)$ & $0.82^{\mathrm{C}}$ \\
\hline \multicolumn{4}{|c|}{ Minimum score (floor effect), $\mathrm{n}(\%)$} \\
\hline FES & $21(13.4)$ & $17(13.4)$ & $1.00^{\mathrm{c}}$ \\
\hline FES-I & $5(3.2)$ & $6(4.7)$ & $0.50^{\mathrm{c}}$ \\
\hline Short FES-I & $22(14.0)$ & $14(11.0)$ & $0.45^{\mathrm{c}}$ \\
\hline \multicolumn{4}{|c|}{ Maximum score (ceiling effect), n (\%) } \\
\hline FES & 0 & 0 & - \\
\hline FES-I & 0 & 0 & - \\
\hline Short FES-I & $1(0.6)$ & 0 & - \\
\hline \multicolumn{4}{|l|}{ Cronbach's $\alpha$} \\
\hline FES & 0.88 & 0.90 & - \\
\hline FES-I & 0.91 & 0.93 & - \\
\hline Short FES-I & 0.82 & 0.86 & - \\
\hline \multicolumn{4}{|c|}{ Mean (minimum-maximum) inter-item correlations } \\
\hline FES & $0.42(0.31-0.56)$ & $0.50(0.36-0.62)$ & - \\
\hline FES-I & $0.40(0.17-0.57)$ & $0.47(0.29-0.68)$ & - \\
\hline Short FES-I & $0.39(0.19-0.53)$ & $0.48(0.29-0.62)$ & - \\
\hline
\end{tabular}

a Cognitively impaired: MMSE score <24; cognitively intact: MMSE score $\geq 24$.

${ }^{b} t$ test to test for differences between the cognitively impaired and cognitively intact group.

${ }^{\mathrm{c}}$ Mann-Whitney U test to test for differences between the cognitively impaired and cognitively intact group.

${ }^{d} \chi^{2}$ to test for differences between the cognitively impaired and cognitively intact group.

${ }^{\mathrm{e}}$ Higher scores indicate increased concerns of falling.
For the total sample and for the groups with or without cognitive impairment, mean scores of the FES, FES-I and Short FES-I are presented according to the categories of the fall risk parameters associated with fear of falling (table 3). For the total group, significant differences $(\mathrm{p}<0.05)$ according to sex for FES and FES-I could be documented. For dizziness, number of medications, falls in the previous year, ADL, timed up-and-go and overall fear of falling, results of all 3 questionnaires were significant.

For the cognitively impaired subgroup, significant differences $(\mathrm{p}<0.05)$ according to dizziness, number of medications, ADL, timed up-and-go and overall fear of falling could be documented 
Table 3. Mean scores and SD of the FES, FES-I and Short FES-I according to background variables for cognitively impaired and cognitively intact older adults ${ }^{\mathrm{a}}$

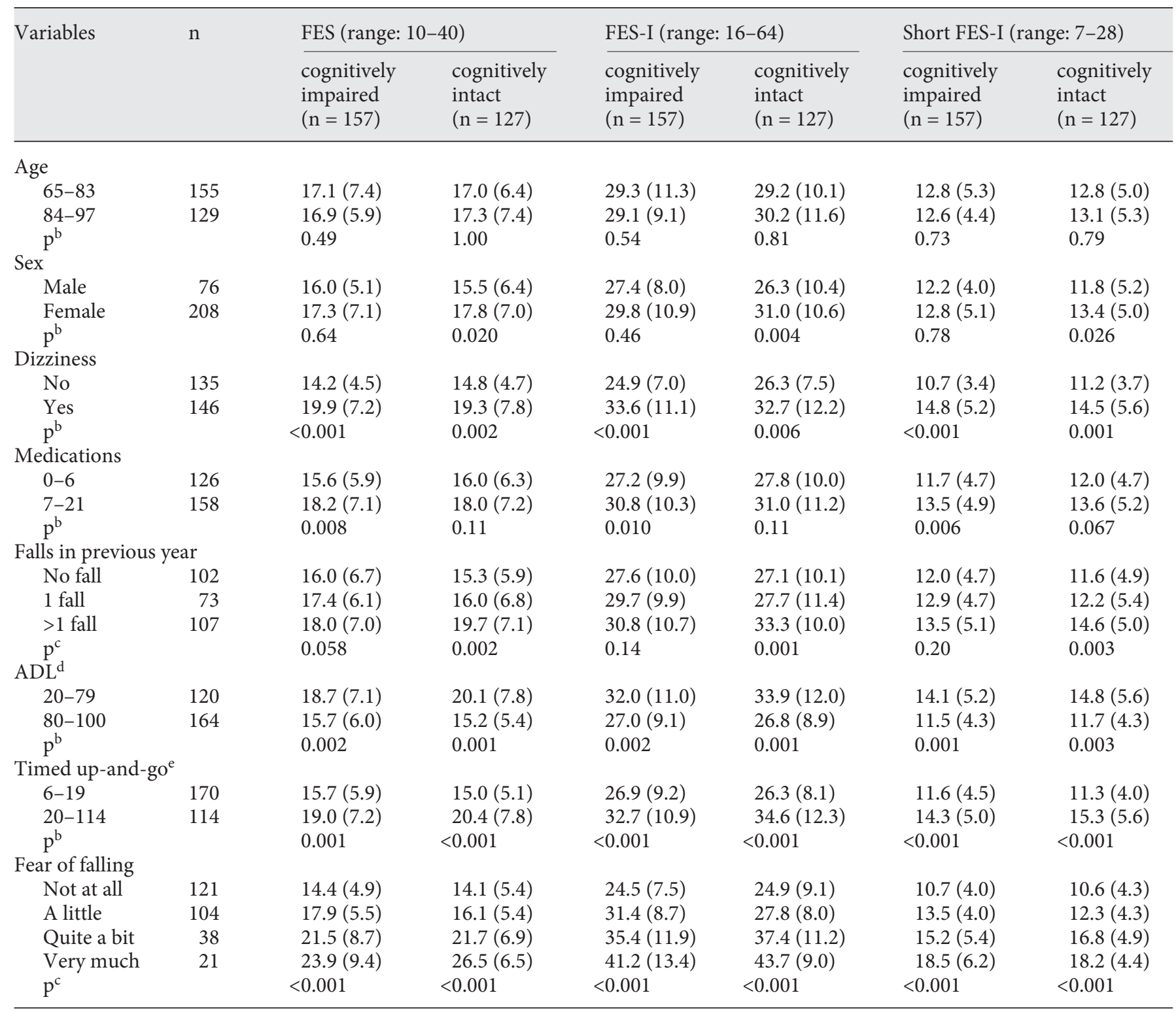

${ }^{a}$ Cognitively impaired: MMSE score $<24$; cognitively intact: MMSE score $\geq 24$.

b Differences tested for background variables with Mann-Whitney U test.

${ }^{c}$ Differences tested for background variables with Kruskal-Wallis test.

${ }^{\mathrm{d}} \mathrm{ADL}=$ activities of daily living, higher values indicate better functional independence.

${ }^{\mathrm{e}}$ Lower values indicate better functional performance.

for results of all 3 questionnaires. For the cognitively intact subgroup significant differences $(\mathrm{p}<0.05)$ according to sex, dizziness, falls in the previous year, ADL, timed up-and-go and overall fear of falling could be documented for results of all 3 questionnaires. Results indicated more concerns in those patients with higher risk of falling as expected. For example, persons with diz- ziness had significantly higher scores than persons without dizziness on all scales. However, age was not related to any of the scale scores. The pattern of scores for the 3 scales were highly similar in cognitively impaired compared to cognitively intact older persons except for history of falls, indicating good construct validity in both subgroups. 
Table 4. Baseline characteristics of the RCT population in the sensitivity to change study (Study 2)

\begin{tabular}{|c|c|c|c|}
\hline Variable & $\begin{array}{l}\text { Intervention } \\
\text { group } \\
(n=67)^{a}\end{array}$ & $\begin{array}{l}\text { Control } \\
\text { group } \\
(n=63)\end{array}$ & $\mathrm{p}$ \\
\hline Age, years & $82.3 \pm 6.5$ & $82.8 \pm 6.9$ & $0.70^{\mathrm{b}}$ \\
\hline \multicolumn{4}{|l|}{ Sex, n (\%) } \\
\hline Male & $17(25.4)$ & $17(27.0)$ & \multirow{2}{*}{$0.83^{\mathrm{c}}$} \\
\hline Female & $50(74.6)$ & $46(73.0)$ & \\
\hline Years of education, $\mathrm{n}$ & $10.9 \pm 3.0$ & $11.1 \pm 2.1$ & $0.69^{\mathrm{b}}$ \\
\hline Cognitive status (MMSE) & $21.9 \pm 2.7$ & $22.1 \pm 3.2$ & $0.75^{\mathrm{b}}$ \\
\hline \multicolumn{4}{|l|}{ Dizziness, $\mathrm{n}(\%)$} \\
\hline No dizziness & $35(52.2)$ & $33(54.1)$ & \multirow{2}{*}{$0.83^{c}$} \\
\hline Dizziness & $32(47.8)$ & $28(45.9)$ & \\
\hline Medical diagnosis, $\mathrm{n}$ & $7.8 \pm 2.6$ & $7.5 \pm 3.0$ & $0.57^{\mathrm{b}}$ \\
\hline Medications, $\mathrm{n}$ & $6.8 \pm 3.1$ & $7.1 \pm 2.8$ & $0.61^{b}$ \\
\hline \multicolumn{4}{|l|}{ Falls in previous year, $\mathrm{n}(\%)$} \\
\hline No falls & $28(42.4)$ & $22(35.5)$ & \multirow{3}{*}{$0.29^{\circ}$} \\
\hline 1 fall & $12(18.2)$ & $19(30.6)$ & \\
\hline$>1$ fall & $26(39.4)$ & $21(33.9)$ & \\
\hline Activities of daily living & $82.1 \pm 15.5$ & $81.6 \pm 13.7$ & $0.85^{\mathrm{b}}$ \\
\hline Timed up-and-go, s & $17.5 \pm 12.5$ & $19.0 \pm 15.7$ & $0.56^{\mathrm{b}}$ \\
\hline \multicolumn{4}{|l|}{ Fear of falling, n (\%) } \\
\hline Not at all & $36(53.6)$ & $31(49.2)$ & \multirow[t]{4}{*}{$0.33^{\mathrm{c}}$} \\
\hline A little & $23(34.4)$ & $18(28.6)$ & \\
\hline Quite a bit & $4(6.0)$ & $10(15.9)$ & \\
\hline Very much & $4(6.0)$ & $4(6.3)$ & \\
\hline \multicolumn{4}{|l|}{ Type of dementia, \% } \\
\hline Alzheimer's disease & 50.0 & 50.0 & \multirow[t]{3}{*}{$0.11^{\mathrm{c}}$} \\
\hline Vascular & 27.4 & 40.0 & \\
\hline Other types & 22.6 & 10.0 & \\
\hline
\end{tabular}

Values are means \pm SD unless otherwise indicated.

a Numbers may not add up to 67 or 63 due to missing values.

${ }^{b} t$ test to test for differences between the cognitively impaired and cognitively intact group.

${ }^{c} \chi^{2}$ to test for differences between the cognitively impaired and cognitively intact group.

\section{Study 2: Sensitivity to Change}

\section{Methods}

\section{Participants}

Patients of geriatric rehabilitation wards with beginning to moderate stage dementia $(n=130)$ who participated in a randomized placebo-controlled trial evaluating a strength and functional exercise intervention aimed to improve motor function (ISRCTN49243245) in Heidelberg, Germany, between 2006 and 2008 [37] were included in this study. The present study (sensitivity to change) provides a selective report detailing only some of the measures used with focus on responsiveness of concerns about falls. Data of this sample were also used in Study 1: Validation. Patients who were screened for participation in the RCT but who did not meet the inclusion criteria (e.g. not residing in the vicinity of the study center or no cognitive impairment) were asked to participate in the validation study. Criteria for participation in this study were similar to those of Study 1, but also included residence in the vicinity of the study center $(<15 \mathrm{~km})$, willingness to participate in a training intervention and diagnosis of beginning to moderate stage dementia confirmed by established diagnostic criteria $[38,39]$ after a positive screening for cognitive impairment by the MMSE (<26 scores). Diagnosis was based on medical history, clinical examination, cerebral imaging, an established neuropsychological test battery (Consortium to Establish a Registry for Alzheimer's Disease) [40] and the Trail Making Test [41] which allowed for the testing of cognitive subperformances necessary for dementia diagnosis. In contrast to Study 1 (patients identified as cognitively impaired by the MMSE), the sample from Study 2 (patients diagnosed with mildto-moderate stage dementia) included different patient groups with respect to cognitive impairment/dementia. Patients meeting the inclusion criteria were randomly assigned to either the intervention group or the control group using an urn design for clinical trial (numbered containers), stratified for gender and location of recruitment (hospitalized vs. others) [42]. The sequence was concealed until interventions were assigned after baseline measurements. A person unrelated to the study performed the randomization procedure and assigned participants to their study group.

The intervention group underwent a high-intensity, progressive strength and functional training for $2 \mathrm{~h}$ twice weekly for 12 weeks, and the control group underwent a low-intensity stretching and ball game exercise for $1 \mathrm{~h}$ twice weekly for 12 weeks. Both groups received the strictly standardized training in small groups (4-6 patients) supervised by a physical exercise trainer [37]. The primary aim of this study was to improve motor performance. The motor training was expected to decrease the fear of falling, as shown in a similar intervention, [43] thus representing an adequate intervention to analyze responsiveness of the studied questionnaires.

The original sample consisted of 130 participants; 17 patients withdrew during the intervention. Statistically, dropouts did not differ significantly (data not shown) from the total sample regarding baseline characteristics as presented in table 4, except for age (81.5 vs. 83.1 years; $\mathrm{p}=0.034$ ). For 1 patient, no data on fear/concerns about falling was documented.

Measures and Data Collection

Measures and data collection correspond to the procedures as described for Study 1 (see above). Assessors were blinded to the patients' group allocation.

\section{Statistical Analysis}

For comparisons between subgroups according to cognitive status, the same statistical analysis was used as in Study 1 (validation study) based on distribution and characteristics of data. To test for sensitivity to change, standard response means (SRMs) were calculated as mean differences between groups divided by the standard deviation of the difference $[44,45]$. SRMs represent established methods for reporting the magnitude of change and are expressed in units of variability without unit of measurement [46]. SRMs of 0.20 are considered small, 0.50 medium and above 0.80 large $[44,46]$. Statistical analyses were performed with SPSS for Windows, Version 17.0 (SPSS Inc., Chicago, Ill., USA). 
Table 5. SRM of the FES, FES-I and Short FES-I for subgroups in patients with beginning to moderate stage dementia

\begin{tabular}{|c|c|c|c|c|}
\hline Groups $^{b}$ & $\mathrm{n}$ & $\begin{array}{l}\text { Mean score } \\
\mathrm{T} 1^{\mathrm{a}}(\mathrm{SD})\end{array}$ & $\begin{array}{l}\text { Mean score } \\
\mathrm{T} 2^{\mathrm{a}}(\mathrm{SD})\end{array}$ & SRM \\
\hline \multicolumn{5}{|l|}{ Total group ${ }^{b}$} \\
\hline FES & 112 & $15.52(5.68)$ & $13.85(4.04)$ & 0.38 \\
\hline FES-I & 112 & $27.21(9.26)$ & $24.63(6.94)$ & 0.36 \\
\hline Short FES-I & 112 & $11.69(4.32)$ & $10.51(3.33)$ & 0.33 \\
\hline \multicolumn{5}{|l|}{ Concerns about falls ${ }^{\mathcal{c}}$} \\
\hline \multicolumn{5}{|l|}{ FES } \\
\hline More concerned & 61 & $19.26(5.21)$ & $15.61(4.41)$ & 0.77 \\
\hline Less concerned & 51 & $11.03(1.20)$ & $11.76(2.17)$ & 0.36 \\
\hline \multicolumn{5}{|l|}{ FES-I } \\
\hline More concerned & 61 & $33.33(8.27)$ & $27.62(7.47)$ & 0.74 \\
\hline Less concerned & 51 & $19.88(2.79)$ & $21.06(4.01)$ & 0.32 \\
\hline \multicolumn{5}{|l|}{ Short FES-I } \\
\hline More concerned & 60 & $14.83(3.48)$ & $11.78(3.43)$ & 0.91 \\
\hline Less concerned & 52 & $8.06(1.21)$ & $9.04(2.56)$ & 0.41 \\
\hline \multicolumn{5}{|l|}{ Cognitive status $\mathrm{d}$} \\
\hline \multicolumn{5}{|l|}{ FES } \\
\hline MMSE <24 & 69 & $15.87(5.79)$ & $13.68(3.57)$ & 0.52 \\
\hline MMSE $\geq 24$ & 43 & $14.95(5.51)$ & $14.14(4.73)$ & 0.18 \\
\hline \multicolumn{5}{|l|}{ FES-I } \\
\hline MMSE <24 & 69 & $27.83(9.54)$ & $24.67(6.17)$ & 0.48 \\
\hline MMSE $\geq 24$ & 43 & $26.21(8.81)$ & $24.58(8.11)$ & 0.21 \\
\hline \multicolumn{5}{|l|}{ Short FES-I } \\
\hline MMSE <24 & 69 & $11.91(4.43)$ & $10.49(3.10)$ & 0.42 \\
\hline MMSE $\geq 24$ & 43 & $11.33(4.16)$ & $10.53(3.72)$ & 0.21 \\
\hline \multicolumn{5}{|l|}{ Intervention status } \\
\hline \multicolumn{5}{|l|}{ FES } \\
\hline Intervention & 57 & $15.60(6.55)$ & $14.30(4.36)$ & 0.28 \\
\hline Control & 55 & $15.44(4.67)$ & $13.40(3.66)$ & 0.52 \\
\hline \multicolumn{5}{|l|}{ FES-I } \\
\hline Intervention & 57 & $27.21(10.64)$ & $25.33(7.47)$ & 0.25 \\
\hline Control & 55 & $27.20(7.67)$ & $23.91(6.33)$ & 0.50 \\
\hline \multicolumn{5}{|l|}{ Short FES-I } \\
\hline Intervention & 57 & $11.67(4.91)$ & $11.00(3.65)$ & 0.18 \\
\hline Control & 55 & $11.71(3.64)$ & $10.00(2.92)$ & 0.51 \\
\hline
\end{tabular}

The intervention showed statistically significant time effects induced by improvement of both groups (total sample: $\mathrm{p}<0.01$; subsample of patients with more concerns: $\mathrm{p}<0.001$ ), but no significant group effect between study groups (total sample: $p=0.78$; subsample of affected patients: $\mathrm{p}=0.35$ ) as calculated by analysis of variance, repeated measures.

${ }^{\text {a }} \mathrm{T} 1$ = baseline measurement; $\mathrm{T} 2=12$-week follow-up after the intervention.

${ }^{\mathrm{b}}$ Higher values indicate more concerns about falling.

${ }^{\mathrm{c}}$ More concerned indicates subgroup with values higher than median at $\mathrm{T} 1$, less concerned indicates subgroup with values lower than median at $\mathrm{T} 1$.

${ }^{d}$ Higher values indicate better cognitive status. MMSE score $<24$ indicates higher level of cognitive impairment and score $\geq 24$ indicates lower level of cognitive impairment.
Results

Participants

A subsample of the total group participated in the intervention and assessments of a randomized placebo-controlled trial $(\mathrm{n}=$ 130). Study groups (intervention vs. control group) in this RCT showed no significant differences at baseline (table 4). Statistical tests showed minor differences between the intervention sample and the total sample with respect to baseline characteristics. The subsample was on average more cognitively impaired (mean MMSE score: 21.98 (range: $15-26$ ) vs. 23.2 ; $\mathrm{p}=0.032$ ) but presented less functional impairment (mean ADL score: 81 vs. $77 ; \mathrm{p}=$ 0.043 .

Sensitivity to Change

Analyses in the subgroups revealed that the FES, FES-I and Short FES-I all showed moderate to good sensitivity to change (table 5). For the total group, an effect of the intervention on concerns about falling was shown $(\mathrm{p}<0.01)$, with highest effects for the subgroup of people who reported more concerns about falling at baseline ( $>$ median score of the total group; $p<0.001)$. All scales tested showed the highest effect sizes for this subgroup indicating excellent sensitivity to change. Despite having the fewest items, the Short FES-I was the most sensitive to change. A comparison between different groups based on MMSE scores (MMSE score $<24$ vs. $\geq 24$ ) revealed a moderate to small effect of cognitive status on responsiveness, with slightly better sensitivity to change in the cognitively impaired group. Participation in the intervention versus control group was associated with different levels of physical training, but did not show substantial influence on the responsiveness of the questionnaires.

\section{Discussion}

Previous studies have documented the excellent reliability and validity of the FES-I and Short FES-I in community-dwelling older adults $[21,23,24]$ and the retest reliability and feasibility of the FES-I in older adults with cognitive impairment [25]. The aim of this study was to investigate validity and internal reliability results for patients with cognitive impairment and to determine the sensitivity to change of the FES, FES-I and Short FES-I in patients with confirmed dementia.

The good to excellent validity and sensitivity to change of the FES, FES-I and the Short FES-I in the present study indicate that these measures are also suitable for application in frail older populations with beginning to moderate stage cognitive impairment or without impairment.

\section{Study 1: Validation}

Several aspects deserve consideration. First, in about $13 \%$ of the population, floor effects were observed in the FES and the Short FES-I versus about $4 \%$ in the FES-I. The latter could be expected since the FES-I had been developed to prevent insensitivity to differences in high 
functioning persons as identified in the original FES. Post-hoc analyses showed that the people who showed floor effects in the FES and Short FES-I differed significantly $(\mathrm{p}<0.05)$ regarding physical functioning, i.e. better ADL and timed up-and-go scores, from persons reporting at least a little concern about falling. However, it may be difficult to completely eliminate floor effects as denial of fear-inducing challenges, such as falls, causes frequent misreporting in patients with repressive coping strategies [47]. On the other hand, absence of fear of falling even in impaired patients may reflect either an adjusted behavior (being careful but not fearful) or, particularly in patients with dementia, a lack of awareness which is a feature commonly characteristic of this condition. Although the floor effects for the Short FES-I were slightly more prevalent in the group with cognitively impaired people, no significant differences between subgroups according to cognitive status were found. No ceiling effect was detected, indicating that even major concerns about falls were adequately covered by all scales, even in the frail population included in this study.

Second, no substantial influence of cognitive impairment was found on the mean scores of the FES, FES-I and Short FES-I and their correlations. Cognitively impaired persons showed marginally lower internal reliability estimates and mean inter-item correlations, but values were comparable to findings in previous studies in persons without cognitive impairment $[21,23,24]$.

Lastly, as there is no objective external standard to measure fear of falling, fall-risk parameters associated with fear of falling have been used to study whether the mean scores of the FES, FES-I and Short FES-I relate to the different subcategories of the parameters. Generally, mean scores of all scales showed trends and significant differences as expected within subcategories of the fallrisk parameters for the total group and the groups according to cognitive status. This confirms previous validation results [21, 23-25] and provides evidence for the construct validity of the Short FES-I in cognitively impaired people. Although fear of falling was higher in persons who reported more falls in the previous year, the association was not statistically significant in the cognitively impaired group. This may be explained by impaired recall of falls and thereby inadequate grouping of these patients according to their history of falls. Although findings were largely similar across the different scales, groups based on age and sex did not show the significant statistical differences expected. The distribution of the sample, e.g. with fairly limited numbers of people under the age of 75 years $(n=34)$, may have contributed to these

Fear of Falling Measures in People with Dementia findings. The results from this study do replicate findings from previous studies which found no association between age and fear of falling [10, 48, 49]. Overall, the results of this validation study are comparable to results of previous validation studies in older nonimpaired persons $[21,23,24]$, indicating that the scales tested are valid for application in frail older persons with and without cognitive impairment.

\section{Study 2: Sensitivity to Change}

To our knowledge, this is the first study to present data on sensitivity to change of fear of falling measures in patients with dementia and different levels of impairment. As documented in a previous review, sensitivity to change has only been addressed in some established fear of falling questionnaires [16]. For those questionnaires reporting on sensitivity to change, observation periods ranged from 3 weeks to 1 year, population samples ranged from high-functioning to needing acute care, and age ranged from 41 to 95 years, all of which limits the comparability of results. In some - but not all - of the studies presenting sensitivity to change data, a standardized intervention targeted to influence fear of falling has been performed [16, 50-52]. The interventions differed from each other which further limited the comparability of results. Effect sizes/SRMs reported in these studies were insufficient to good, ranging from 0.00 to 1.50 . Of the self-efficacy measures, the original FES was found to have adequate sensitivity to change. A single item fear of falling question had limited evidence for its sensitivity to change, and none of the other multi-item measures of fear of falling had evidence of sensitivity to change. No data related to patients with cognitive impairment or dementia, respectively, were reported [16]. As with reliability measures, sensitivity to change measurements suffer from a methodological problem: do insufficient effect sizes indicate a methodological flaw in the questionnaire used, or alternatively an insufficient effect of intervention? To prevent methodological inconsistency in the presented RCT to test sensitivity to change, we examined general effects of strictly standardized intervention, influence of cognitive impairment and subsamples with expected higher responsiveness. All 3 scales adequately assessed the change over time achieved by the standardized intervention. Subjects in this study took part in a standardized, supervised group training for 2-4 h per week focusing on motor training such as strength and functional or stretching exercises [37]. The intervention used was unspecific for effects on fear of falling, so data obtained would be expected to be lower bound estimates 
of responsiveness. During the training period, fear of falling significantly decreased in both groups $(p<0.001)$, but did not show significant differences in betweengroup comparisons.

The level of sensitivity to change depended on the groups compared. A subanalysis (table 5) was performed excluding patients who showed little or no fear of falling. Such an analysis minimizes insufficient effects of an intervention by preventing floor effects in patients with no fear. This methodological approach revealed good to excellent sensitivity to change in all tested questionnaires. The subanalysis of influence of cognitive status (MMSE) and study group (intervention vs. control) showed low responsiveness, indicating low influence of such parameters on change of concerns about falling in this intervention trial.

Surprisingly, the measure with the lowest number of items, i.e. the Short FES-I, achieved the highest peak sensitivity to change. In all tests, mean sensitivity to change was comparable for all scales. Sensitivity to change in patients with more severe cognitive impairment as screened by the MMSE was even slightly better, indicating that all measurements may be suitable to assess fear of falling in patients with confirmed mild to moderate dementia.

\section{Limitations}

In the present study, all participants reported on the items of the FES-I and, subsequently, the 7 and 10 items of the Short FES-I and FES were derived from the FES-I. This was done to minimize the assessment burden of the participants and health care providers. Reporting on the remaining items of the FES-I may, however, have influenced the answers regarding the 7 and 10 items of the Short FES-I and FES.

In the observational study (Study 1), cognitive impairment was rated according to an established screening instrument (MMSE). As with other screening instruments, results may have been influenced by parameters such as education or age which affect specificity and sensitivity of grouping (impaired vs. nonimpaired). As for most cases in clinical and research settings, such a rating would be adequate to identify persons suspected of having cognitive impairment and to represent an established assessment procedure. Therefore, a time- and resourcedemanding comprehensive assessment to diagnose dementia or types of dementia was not applied in the observational study.

The 2 samples of the validation (Study 1) and sensitivity to change study (Study 2) differ with respect to the confirmation of the dementia diagnosis which was only performed in Study 2. Results of Study 1 are limited to a sample identified as cognitively impaired by an established screening test using a lower cutoff value (cutoff $=24$ ) to improve specificity of the screening tests. Results obtained in such a sample may deviate from results of people screened by a higher cutoff value for MMSE to increase sensitivity with a confirmed dementia diagnosis. Assessments have been documented by interviews in patients with beginning to moderate stage dementia and cognitive impairment. Assessments of concerns about falling may be limited in more affected patients.

\section{Conclusion}

The results of the study show that the interview-administered FES, FES-I and Short FES-I are valid, reliable and responsive measures of concerns about falling in both cognitively intact and cognitively impaired geriatric patients or patients with beginning to moderate stage dementia. However, if time allows and respondents are able to complete the interview, application of the 16-item FES-I may be desirable to prevent floor effects. All scales showed good sensitivity to change, but the best evidence was shown in the Short FES-I.

\section{Acknowledgements}

We kindly thank the persons who participated in the studies and D. Schrank for his assistance in the data handling. The School for Public Health and Primary Care - CAPHRI and the Faculty of Health, Medicine and Life Sciences of Maastricht University are acknowledged for the support that enabled G.A.R. Zijlstra's stay in Heidelberg during the preparation of the manuscript.

\section{Funding}

This study was performed within the Prevention of Falls Network Europe and supported by a European Committee grant under the Fifth Framework Program QLK6-CT-200-02705 (www. profane.eu.org) and funded by the Robert Bosch Foundation, Stuttgart, the Dietmar Hopp Stiftung, St. Leon-Rot and the Landesstiftung Baden-Württemberg, Stuttgart. 


\section{References}

1 Gillespie LD, Robertson MC, Gillespie WJ, 16 Jorstad EC, Hauer K, Becker C, Lamb SE: Lamb SE, Gates S, Cumming RG, Rowe BH: Interventions for preventing falls in older people living in the community. Cochrane Database Syst Rev 2009: CD007146.

-2 Kannus P, Parkkari J, Koskinen S, Niemi S, Palvanen M, Jarvinen M, Vuori I: Fall-induced injuries and deaths among older adults. JAMA 1999;281:1895-1899.

3 Stel VS, Smit JH, Pluijm SM, Lips P: Consequences of falling in older men and women and risk factors for health service use and functional decline. Age Ageing 2004;33:5865.

4 Vellas BJ, Wayne SJ, Romero LJ, Baumgartner RN, Garry PJ: Fear of falling and restriction of mobility in elderly fallers. Age Ageing 1997;26:189-193.

5 Tinetti ME, Speechley M, Ginter SF: Risk factors for falls among elderly persons living in the community. N Engl J Med 1988;319: 1701-1707.

6 Friedman SM, Munoz B, West SK, Rubin GS, Fried LP: Falls and fear of falling: which comes first? A longitudinal prediction model suggests strategies for primary and secondary prevention. J Am Geriatr Soc 2002; 50:1329-1335.

7 Franzoni S, Rozzini R, Boffelli S, Frisoni G, Trabucchi M: Fear of falling in nursing home patients. Gerontology 1994;40:38-44.

-8 Zijlstra GA, van Haastregt JC, van Eijk JT, van Rossum E, Stalenhoef PA, Kempen GI: Prevalence and correlates of fear of falling, and associated avoidance of activity in the general population of community-living older people. Age Ageing 2007;36:304-309.

9 Bruce DG, Devine A, Prince RL: Recreational physical activity levels in healthy older women: the importance of fear of falling. J Am Geriatr Soc 2002;50:84-89.

$\checkmark 10$ Maki BE: Gait changes in older adults: predictors of falls or indicators of fear? J Am Geriatr Soc 1997;45:313-320.

11 Morris J, Rubin E, Morris E, Mandel S: Senile dementia of the Alzheimer's type: an important risk factor for serious falls. J Gerontol 1987;42:412-417.

$\checkmark 12$ Buchner D, Larson E: Falls and fractures in patients with Alzheimer-type dementia. JAMA 1987;257:1492-1495.

13 Lord S, Sherrington C, Menz H: Falls in Older People. Cambridge, Cambridge University Press, 2001.

- 14 Baker B, Duckworth T, Wilkes E: Mental state and other prognostic factors in femoral fractures of the elderly. J Royal Coll Gen Pract 1978;28:557-559.

$\checkmark 15$ Hauer K, Becker C, Lindemann U, Beyer N: Effectiveness of physical training on motor performance and fall prevention in cognitively impaired older people: a systematic review. Am J Phys Med Rehabil 2006;85:847857.

Fear of Falling Measures in People with Dementia
Measuring the psychological outcomes of falling: a systematic review. J Am Geriatr Soc 2005;53:501-510.

17 Afken CL, Lach HW, Birge SJ. The prevalence and correlates of fear of falling in elderly persons living in the community. Am J Public Health 1994;84:565-570.

18 Liddle J, Gilleard C: The emotional consequences of falls for older people and their families. Clin Rehabil 1995;9:110-114.

19 McKee KJ, Orbell S, Austin CA et al: Fear of falling, falls efficacy and health outcomes in older people following hip fracture. Disabil Rehabil 2002;6:327-333.

20 Tinetti ME, Richman D, Powell L: Falls efficacy as a measure of fear of falling. J Gerontol 1990;45:P239-P243.

21 Yardley L, Beyer N, Hauer K, Kempen G, Piot-Ziegler C, Todd C: Development and initial validation of the Falls Efficacy ScaleInternational (FES-I). Age Ageing 2005;34: 614-619.

22 Lachman ME, Howland J, Tennstedt S: Fear of falling and activity restriction: the survey of activities and fear of falling in the elderly (SAFE). J Gerontol B Psychol Sci Soc Sci 1998;53:S43-S50.

23 Kempen GIJM, Todd CJ, van Haastregt JCM, Zijlstra GAR, Beyer N, Freiberger E, Hauer K, Piot-Ziegler C, Yardley L: Cross-cultural validation of the Falls Efficacy Scale International (FES-I) in older people: results from Germany, the Netherlands and the UK were satisfactory. Disabil Rehabil 2007;29:155162.

24 Kempen GI, Yardley L, van Haastregt JC, Zijlstra GA, Beyer N, Hauer K, Todd C: The Short FES-I: a shortened version of the Falls Efficacy Scale-International to assess fear of falling. Age Ageing 2008;37:45-50.

25 Hauer K, Yardley L, Beyer N, Kempen G, Dias N, Campbell M, Becker C, Todd C: Validation of the Falls Efficacy Scale and Falls Efficacy Scale International in geriatric patients with and without cognitive impairment: results of self-report and interviewbased questionnaires. Gerontology 2010;56: 190-199.

-26 Denkinger M, Igl W, Coll-Planas L, Nikolaus T, Bailer S, Bader A, Jamour M: Practicality, validity and sensitivity to change of fear of falling self-report in hospitalised elderly - a comparison of four instruments. Age Ageing 2009;38:108-112.

27 Folstein MF, Folstein SE, McHugh PR: 'Mini-mental state'. A practical method for grading the cognitive state of patients for the clinician. J Psychiatr Res 1975;12:189-198.

28 Skelton D, Becker C, Lamb S, Close J, Zijlstra W, Yardley L, Todd C: Prevention of Falls Network Europe: a thematic network aimed at introducing good practice in effective falls prevention across Europe. Eur J Ageing 2004;1:89-94.
29 Maki BE, Holliday PJ, Topper AK: Fear of falling and postural performance in the elderly. J Gerontol 1991;46:M123-M131.

-30 Mahoney F, Brody E: Assessment of older people: self-maintaining and instrumental activity of daily living. Gerontologist 1969;9: 179-186.

-31 Podsiadlo D, Richardson S: The timed 'Up \& Go': a test of basic functional mobility for frail elderly persons. J Am Geriatr Soc 1991; 39:142-148.

32 Guidelines for the prevention of falls in older persons. American Geriatrics Society, British Geriatrics Society and American Academy of Orthopedic Surgeons Panel of Falls Prevention. J Am Geriatr Soc 2001;49:664672.

33 Todd C, Skelton D: What are the main risk factors for falls among older people and what are the most effective interventions to prevent theses falls? Copenhagen, WHO Regional Office for Europe (Health Evidence Network Report). http://Euro.WHO.int/ document/E82552.pdf (accessed May 15, 2010).

34 Masud T, Morris RO: Epidemiology of falls. Age Ageing 2001;30(Suppl 4):3-7.

35 Rubenstein LZ: Falls in older people: epidemiology, risk factors, and strategies for prevention. Age Ageing 2006;35(Suppl 2):37-41.

36 George D, Mallery P: 2007. SPSS for Windows Step by Step - A Simple Guide and Reference, 14.0 Update. Boston, Pearson/Allyn and Bacon, 2007.

37 Hüger D, Zieschang T, Schwenk M, Oster P, Becker C, Hauer K: Designing studies on the effectiveness of physical training in patients with cognitive impairment. Z Gerontol Geriatr 2009;42:11-19.

38 McKhann G, Drachman D, Folstein M, Katzman R, Price D, Stadlan EM: Clinical diagnosis of Alzheimer's disease: report of the NINCDS-ADRDA Work Group under the auspices of Department of Health and Human Services Task Force on Alzheimer's Disease. Neurology 1984;34:939-944.

39 Roman GC, Tatemichi TK, Erkinjuntti T, Cummings JL, Masdeu JC, Garcia JH, Amaducci L, Orgogozo JM, Brun A, Hofman A: Vascular dementia: diagnostic criteria for research studies. Report of the NINDS-AIREN International Workshop. Neurology 1993; 43:250-260.

40 Morris JC, Mohs RC, Rogers H, Fillenbaum G, Heyman A: Consortium to establish a registry for Alzheimer's disease (CERAD) clinical and neuropsychological assessment of Alzheimer's disease. Psychopharmacol Bull 1988;24:641-652.

41 Oswald WD, Fleischmann U: Nürnberger Altersinventar (NAI). Göttingen, Hogrefe, 1995.

$-$

Gerontology 2011;57:462-472 
42 Wei LJ: A class of designs for sequential clinical trials. J Am Stat Assoc 1977;72:382-386.

43 Hauer K, Rost B, Rütschle K, Opitz H, Specht N, Bärtsch P, Oster P, Schlierf G: Exercise training for rehabilitation and secondary prevention of falls in geriatric patients with a history of injurious falls. J Am Geriatr Soc 2001;49:10-20.

44 Kazis LE, Anderson JJ, Meenan RF: Effect sizes for interpreting changes in health status. Med Care 1989;27:S178-S189.

-45 Katz JN, Larson MG, Phillips CB, Fossel AH, Liang $\mathrm{MH}$ : Comparative measurement sensitivity of short and longer health status instruments. Med Care 1992;30:917-925.
46 Cohen J: A power primer. Psychol Bull 1992; 112:155-159.

47 Hauer K, Tremmel A, Schuler M, Pfisterer M, Oster P: Effect of repressive coping in geriatric patients' report. Z Geriatr Gerontol 2010; 18:25-31.

48 Cumming RG, Salkeld G, Thomas M, Szonyi G: Prospective study of the impact of fear of falling on activities of daily living, SF-36 scores, and nursing home admission. J Gerontol A Biol Sci Med Sci 2000;55:M299M305.

49 Howland J, Peterson EW, Levin WC, Fried L, Pordon D, Bak S: Fear of falling among the community-dwelling elderly. J Aging Health 1993;5:229-243.
50 Cameron ID, Stafford B, Cumming RG, Birks C, Kurrle SE, Lockwood K, Quine S, Finnegan T, Salkeld G: Hip protectors improve falls self-efficacy. Age Ageing 2000;29: 57-62.

51 Petrella RJ, Payne M, Myers A, Overend T, Chesworth B: Physical function and fear of falling after hip fracture rehabilitation in the elderly. Am J Phys Med Rehabil 2000;79: 154-160.

52 Steadman J, Donaldson N, Kalra L: A randomized controlled trial of an enhanced balance training program to improve mobility and reduce falls in elderly patients. J Am Geriatr Soc 2003;51:847-852. 